\title{
Grupo de Pesquisa em Redes de Computadores, Sistemas Distribuídos e Multimídia
}

\author{
Rommel Wladimir de Lima ${ }^{1}$, Marcos Vinicius de Andrade Lima ${ }^{1}$ \\ ${ }^{1}$ Programa de Pós-graduação em Ciência da Computação (PPgCC) - Universidade do \\ Estado do Rio Grande do Norte (UERN) \\ BR 110, Km 48, Rua Prof. Antônio Campos, Costa e Silva, Mossoró-RN, Brasil \\ rommelwladimir@uern.br, marcos.engsoft@gmail.com
}

O grupo foi criado inicialmente para área de Redes de Computadores e Sistemas Distribuídos, mas atualmente atua fortemente na área de tecnologias educacionais com os seguintes temas de interesse, não exclusivos: Ambiente Virtual de Aprendizagem; Aprendizagem Colaborativa Baseada na Web; Aprendizagem com Suporte de Computador; Educação a Distância; Objetos de Aprendizagem; Televisão Digital Interativa; Software Educativo; Formação Permanente e outros temas relacionados com tecnologias educacionais, inclusive Educação Especial. Contando com a participação de pesquisadores da Universidade do Estado do Rio Grande do Norte (UERN), Universidade Federal Rural do Semi-Árido (UFERSA) e Universidade Federal do Rio Grande do Norte (UFRN). Recebendo fomentos da FAPERN, CAPES e CNPq.

Para dar suporte às suas atividades, o grupo conta com a participação de diversos pesquisadores, listados na Tabela 1:

Tabela 1. Relação de Pesquisadores do Grupo

\begin{tabular}{|c|c|c|c|}
\hline  & $\begin{array}{l}\text { Rommel Wladimir de Lima } \\
\text { Carla Katarina de Monteiro Marques } \\
\text { Aquiles M. Filgueira Burlamaqui } \\
\text { Alysson Mendes de Oliveira } \\
\text { João de Deus Lima } \\
\text { Sebastiao Emidio Alves Filho }\end{array}$ & 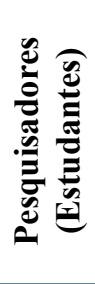 & $\begin{array}{l}\text { Flávio Gustavo Tomás Lima de Freitas } \\
\text { José Osvaldo Mesquita Chaves } \\
\text { Karl Hansimuller Alelaf Ferreira } \\
\text { Marcos Vinicius de Andrade Lima } \\
\text { Maria das Graças Pereira da Silva } \\
\text { Selma Márcia Pontes Teixeira Rocha } \\
\text { Thiago Reis da Silva }\end{array}$ \\
\hline
\end{tabular}

As principais pesquisas atualmente trabalhadas pelo grupo são:

- Desenvolvimento de repositório de objetos de aprendizagem para OBA-MC e de aplicação interativa para facilitar o aprendizado mediado pela TV Digital;

- Construção de laboratório virtual para o ensino de redes de computadores e ferramenta para auxiliar atividades práticas em disciplinas de programação;

- Proposta de fomento ao uso das TICs nos cursos de graduação da UERN.

Em 2013 o grupo teve trabalhos aceitos em diversos eventos, entre eles:

- $24^{\circ}$ Simpósio Brasileiro de Informática na Educação (SBIE)

- VIII Conf. Latino-Americana Objetos e Tecnologias de Aprendizagem (LACLO)

- II Workshop de Desafios da Computação Aplicada à Educação (DesafIE!)

- XXI Workshop sobre Educação em Computação (WEI)

- XXI Ciclo de Palestras sobre Novas Tecnologias na Educação (CPNTE)

- X Congresso Brasileiro de Ensino Superior a Distância (ESUD) 